\title{
Limiting spectral distribution of large dimensional random matrices of linear processes
}

\author{
Zahira Khettab \\ University of Tlemcen, Department of Mathematics, Statistics and Random Modeling Laboratory, Tlemcen, \\ Algeria
}

\begin{abstract}
The limiting spectral distribution (LSD) of large sample radom matrices is derived under dependence conditions. We consider the matrices $X_{N} T_{N} X_{N}^{\prime}$, where $X_{N}$ is a matrix $(N \times n(N))$ where the column vectors are modeled as linear processes, and $T_{N}$ is a real symmetric matrix whose LSD exists. Under some conditions we show that, the LSD of $X_{N} T_{N} X_{N}^{\prime}$ exists almost surely, as $N \rightarrow \infty$ and $n(N) / N \rightarrow c>0$. Numerical simulations are also provided with the intention to study the convergence of the empirical density estimator of the spectral density of $X_{N} T_{N} X_{N}^{\prime}$.
\end{abstract}

\section{Introduction}

Let $X_{(t)}=\left(X_{1 . t}, X_{2 . t}, \ldots, X_{N . t}\right)^{\prime}, t=1,2, \ldots, n$, where $\left\{X_{i . j}, 1 \leq i \leq N, 1 \leq j \leq n\right\}$ is an array of real random variables, and ()$^{\prime}$ indicates the transpose of a vector or matrix. In the theory of large dimensional random matrices, the sample covariance matrix is defined as

$$
X_{N} X_{N}^{\prime}
$$

where

$$
X_{N}=\frac{1}{\sqrt{N}}\left(X_{(1)}, X_{(2)}, \ldots, X_{(n)}\right) .
$$

Sample covariance matrices are very important in multivariate statistical inference since many test statistics are defined by their eigenvalues or functionals. The spectral theory of large dimensional sample covariance matrices also finds wide application in signal detection and array processing. Under the assumption that all variables $X_{i . j}$ are independent and identically distributed (i.i.d.), the spectral analysis of large dimensional covariance matrices has been active since the pioneering work of Marcenko and Pastur (1967) who state that if $N \rightarrow \infty$ such that $\frac{n(N)}{N} \rightarrow c>0$, then the empirical spectral distribution (ESD) of the covariance matrix $X_{N} X_{N}^{\prime}$, converges to a non-random distribution. This limiting spectral distribution (LSD), named Marcenko-Pastur distribution of index $c$ has a density function

$$
f(x)=\frac{1}{2 \pi c x} \sqrt{(b-x)(x-a)}, \quad a \leq x \leq b
$$

Email address: khettab.zahira@yahoo.fr (Zahira Khettab) 
with $a=(1-\sqrt{c})^{2}$ and $b=(1+\sqrt{c})^{2}$ defining the support interval and has a point mass $1-1 / c$ at the origin if $c>1$. Extensions can be found in the work of Wachter (1980) and Yin (1986) who established the almost sure results and Jin et al. (2014) generalized it to auto-covariance matrix.

To relax the independence condition on the entries of $X_{(t)}$, Silverstein (1995) considered the case of $X_{(t)}=T^{\frac{1}{2}} Y_{(t)}$, where $T$ is a non-negative definite matrix and $Y_{(t)}$ consists of i.i.d. entries. Some further investigation on this model can be found in Silverstein and Bai (1995) for strong convergence of the ESD of the sample covariance matrices, in Silverstein and Bai (1999) for the spectrum separation. Furthermore, Boutet de Monvel and Khorunzhy (1998), Wang and Paul (2014), Merlevede and Peligrad (2016), and Bhattacharjee and Bose (2016), among others, treated large covariance matrices based on correlated random variables. Many important classes of high-dimensional random matrcies, particularly those arising in signal processing, economics and finance, have the feature that in addition to the dimensional correlation, the observations are correlated in time.

Define the ESD of any $(N \times N)$ symmetric matrix $M$ as

$$
F^{M}(x)=\frac{1}{N} \sum_{i=1}^{N} 1_{\left(\lambda_{i} \leq x\right)},
$$

where $1_{A}$ denotes the indicator function of the set $A$, and $\lambda_{1}, \lambda_{2}, \ldots, \lambda_{N}$ are the eigenvalues of $M$.

Note that, $F$ the LSD of the sample covariance matrices can in general only be given in terms of a non-linear equation for its Stieltjes transform, that is defined by

$$
m_{F}(z)=\int \frac{1}{\lambda-z} d F(\lambda), \quad z \in \mathbb{C}^{+} \equiv\{z \in \mathbb{C}: \mathfrak{I m} z>0\},
$$

where $\mathfrak{I m} z$ is the imaginary part of $z$.

Conversely, the distribution $F$ can be obtained from its Stieltjes transform $m_{F}$ via the inversion formula (Theorem B.8 (2014)), which states that

$$
F([a, b])=\frac{1}{\pi} \lim _{\varepsilon \rightarrow 0^{+}} \int_{a}^{b} \mathfrak{I m} m_{F}(x+i \varepsilon) d x,
$$

where $a, b$ are continuity points of $F$. Stieltjes transforms play an important role in deriving LSDs. They have also been useful in studying rates of convergence.

We consider the matrix

$$
X_{N} T_{N} X_{N}^{\prime}
$$

where $X_{N}$ is a matrix $(N \times n(N))$ where the column vectors are modeled as linear processes, and $T_{N}$ is an $(n(N) \times n(N))$ real symmetric matrix. We work in the framework of the particularly high-dimensional model where the dimension $n=n(N)$ increases proportionately with the sample size $N$, so that $N \rightarrow \infty$ and $\frac{n(N)}{N} \rightarrow c>0$.

Our aim in this note is to obtain the Marcenko-Pastur type result for random matrix of the form (1), in the case where there is dependence within the columns of $X_{N}$. More precisely, the $t$-th column of $X_{N}$ is given by the linear process form described below. In this way we prove under some conditions that the LSD of (1) exists almost surely. Numerical simulations enlighten on the impact of different parameters, on the convergence rate of density estimator of the spectral density of (1).

The paper is organized as follows. Section 2 provides the main theorem. In Section 3 we present results of numerical simulations. Section 4 is dedicated to the description of details about the programs used to estimate the models. The conclusions of the present study are discussed in Section 5. 


\section{Main result}

To obtain the result in the case of a general linear process for the random matrices (1), consider the following assumptions.

Assumption A1. $X_{(t)}$, the $(N \times 1)$ dimensional $t$-th column vector of the data matrix $X_{N}$, satisfies

$$
X_{(t)}=A_{N} X_{(t-1)}+\varepsilon_{t}, \quad \forall t>1,
$$

where $A_{N}$ is an $(N \times N)$ real matrix with $\sup _{N} \lambda_{\text {max }}\left(A_{N}^{\prime} A_{N}\right)<1$, LSD of $A_{N}$ exists, and $\varepsilon_{t}$ is given in (A2).

Assumption A2. $\left\{\varepsilon_{t}=\left(\varepsilon_{1 . t}, \varepsilon_{2 . t}, \ldots, \varepsilon_{N . t}\right)^{\prime}\right\}$ are $(N \times 1)$ independently distributed random vectors whose entries are also independent with mean 0 , variance $\sigma^{2}>0, \sup _{i j} E\left(\varepsilon_{i . j}\right)^{4}<\infty$ and for some $\delta \in(0,2]$,

$$
\lim _{N \rightarrow \infty} \frac{1}{N n(N)} \sum_{t=1}^{n(N)} \sum_{i=1}^{N} E\left(\left|\varepsilon_{i . t}\right|^{2+\delta} 1_{\left(\left|\varepsilon_{i . t}\right|>\sqrt{n}\right)}\right)=0 .
$$

Assumption A3. $T_{N}$ is a sequence of norm bounded $(n(N) \times n(N))$ real symmetric matrices whose LSD exists. For each $k \geq 0$, let the sequence of $(n(N) \times n(N))$ matrix $P_{k, N}$ whose $k$-th upper diagonal is 1 and 0 otherwise. Note that $P_{0, N}=I_{N}$ the identity matrix of order $N$. Suppose average trace of any finite order polynomial in $\left\{T_{N}, P_{k, N}, P_{k, N}^{\prime}: k \geq 0\right\}$ converges as $N \rightarrow \infty$.

Our main result is the following theorem.

Theorem 1. Under (A1), (A2) and (A3), as $N \rightarrow \infty$ and $\frac{n(N)}{N} \rightarrow c>0$, the LSD of $X_{N} T_{N} X_{N}^{\prime}$ exists almost surely and it can be described in terms of polynomials in some free independent variables.

The above LSD can be described in terms of freely independent variables, for details see Sections 2 and 3 of Bhattacharjee and Bose (2016).

Proof. We have

$$
X_{(t)}=\sum_{k=0}^{t-1}\left(A_{N}\right)^{k} \varepsilon_{t-k}, \quad \forall t \geq 1 .
$$

First define two processes, for $q \geq 0$

$$
\tilde{X}_{(t)}=\sum_{k=0}^{q}\left(A_{N}\right)^{k} \varepsilon_{t-k}, \quad \forall t \geq 1
$$

and

$$
\hat{X}_{(t)}=\sum_{k=0}^{q}\left(A_{N}\right)^{k} \hat{\varepsilon}_{t-k}, \quad \forall t \geq 1
$$

where

$$
\hat{\varepsilon}_{i . t}=\varepsilon_{i . t} 1_{\left(\left|\varepsilon_{i . t}\right|<\sqrt{n}\right)}-E\left(\varepsilon_{i . t} 1_{\left(\left|\varepsilon_{i . t}\right|<\sqrt{n}\right)}\right) .
$$

By Theorem 3.1 of Bhattacharjee and Bose (2016), the almost sure LSD of $\hat{X}_{N} T_{N} \hat{X}_{N}^{\prime}$ exists for each $0 \leq q<\infty$. For a fixed $q$ let us denote this LSD by $F_{q}$. Let $L(.,$.$) be the Lévy$ metric between two distribution functions. Then

$$
\lim _{N \rightarrow \infty} L\left(F^{\hat{X}_{N} T_{N} \hat{X}_{N}^{\prime}}, F_{q}\right)=0, \quad \forall q \geq 0 .
$$


Now by Corollary 3.1 of Bhattacharjee and Bose (2016), there is a probability distribution function $F$ such that

$$
\lim _{q \rightarrow \infty} L\left(F_{q}, F\right)=0 .
$$

After renaming $\psi_{j}=\left(A_{N}\right)^{j}$ and $P_{j-j^{\prime}+k, N}=P_{j, N} T_{N} P_{j^{\prime}+k, N}$ for all $j, j^{\prime}, k$ and $N$ in the proof of the Remark 3.1 (a) and (b) of Bhattacharjee and Bose (2016), it is immediate that

$$
\lim _{N \rightarrow \infty} L\left(F^{\tilde{X}_{N} T_{N} \tilde{X}_{N}^{\prime}}, F^{\hat{X}_{N} T_{N} \hat{X}_{N}^{\prime}}\right)=0, \quad \forall q \geq 1 .
$$

Also by Corollary 3.1 of Bhattacharjee and Bose (2016), it follows that

$$
\lim _{q \rightarrow \infty} \lim _{N \rightarrow \infty} L\left(F^{X_{N} T_{N} X_{N}^{\prime}}, F^{\tilde{X}_{N} T_{N} \tilde{X}_{N}^{\prime}}\right)=0 .
$$

Combining eqs. (2) to (5) the proof of the theorem is complete.

\section{Numerical Simulations}

In this section, we illustrate the behavior of the empirical density estimator of the spectral density of large random matrices $X_{N} T_{N} X_{N}^{\prime}$, and identify the density function of the limit law by numerical simulations, in the special case of the assumption (A1): $A_{N}=\rho I_{N}$ where $|\rho|<1$, and (A3): The sequence of matrices $T_{N}$ is diagonal with real entries.

From Silverstein and Choi (1995), recall the density of the limit law, for all $x \in \mathbb{R}-\{0\}$, $F$ the LSD has a continuous derivative $f$ on $\mathbb{R}-\{0\}$ given by

$$
f(x)=(1 / \pi) \mathfrak{I m} m_{0}(x),
$$

where $m_{0}(x)$ is given by Stieltjes transform $m_{F}(z)$ as

$$
\lim _{z \rightarrow x} m_{F}(z)=m_{0}(x)
$$

where $z=x+i y$.

We treat two cases:

- Case 1: $T_{N}$ is the identity matrix $(n \times n),(n=n(N))$.

- Case 2: $T_{N}$ is a diagonal matrix: $T_{N}=\operatorname{diag}\left(\tau_{1}, \ldots, \tau_{n}\right), \tau_{i}$ are real rv's.

To analyze the behavior of the empirical estimator we vary dimension $c$, parameter $\rho$ (autoregressive parameter), sample size $N$ and eigenvalues of $T_{N}$. The values of autoregressive parameter $\rho$ reflect the dependence between rv's $X_{i . j}$. Values of parameter $\rho$ near 0 mean near independence and values near 1 mean strong dependence. The values of the dimension $c$ and the number of eigenvalues of $T_{N}$ have an effect on the shape of the limit density function and on convergence rate of the estimator as well. The variability of these parameters may also indicates particular choices that stabilize the estimator, giving hence a good performance.

Case 1 ( $T_{N}$ identity matrix). Plots of the spectral density of $X_{N} T_{N} X_{N}^{t}$ and empirical density given by Stieltjes transform method obtained in this case, for dimension $c=1$, two values of autoregressive parameter $\rho$ (weak and strong dependence) and different sample sizes $N$ are displayed in Figure1. Similarly, for $c=2$ and $c=4$, see Figure 2 and Figure 3 respectively, for a graphical representation of the densities one obtains for different values of $\rho$ and $N$. 

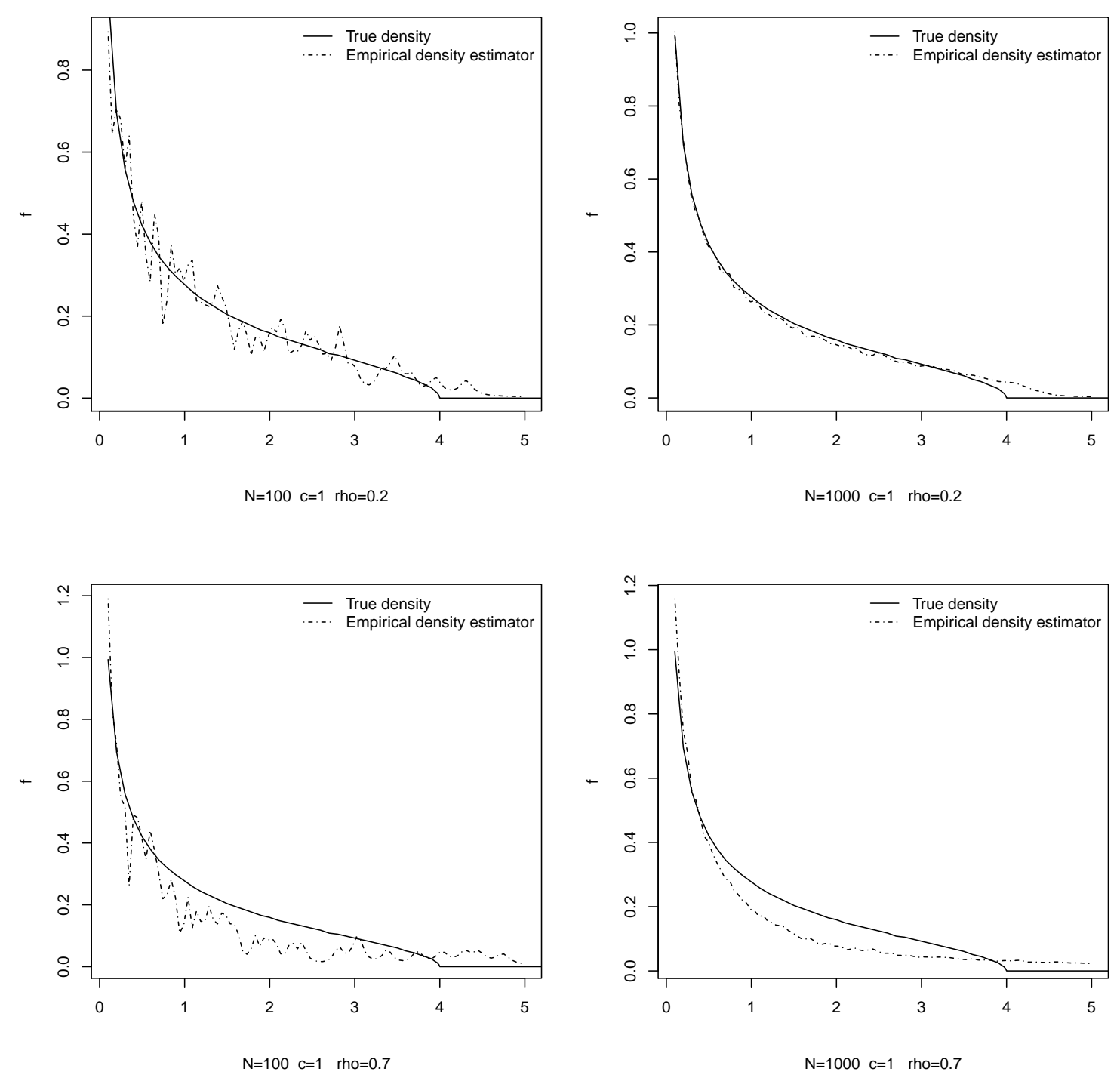

Figure 1: Densities of the limit law and empirical estimator for $c=1$ and $\rho=0.2, \rho=0.7$ with $N=100, N=1000$

Case 2 ( $T_{N}$ diagonal matrix). Figure 4 illustrates a representation of the densities in the case of the diagonal matrix $T_{N}$ with the two eigenvalues 1.5 and 4 with the multiplicities 15 and 35 respectively for the sample size $N=500$, provided that $c=0.1$. The same, Figure 5 gives the representations with the following data, $T_{N}$ diagonal matrix of three eigenvalues 1 , 3 and 10 with the multiplicities 20,40 and 40 respectively for $N=500$, with $c=0.2$, and in Figure 6, $T_{N}$ diagonal matrix of four eigenvalues $0.5,2,5$ and 13 with the multiplicities 30, 10, 20 and 40 respectively for $N=500$, with $c=0.2$. The eigenvalues will have double multiplicities for the sample size $N=1000$.

The results in Table 1 shows that small $L_{1}$-errors values are attained for $N=1000$. As we note that in the case of 3 eigenvalues and $N=1000$, we have the same values for the two autoregressive parameter values.

\section{Description of the estimation program}

Estimates carried out in the all of graphs are based on the method of the Steltjes transform using the $\mathrm{R}$ software. The code for the simulations is given in the online appendix. To 

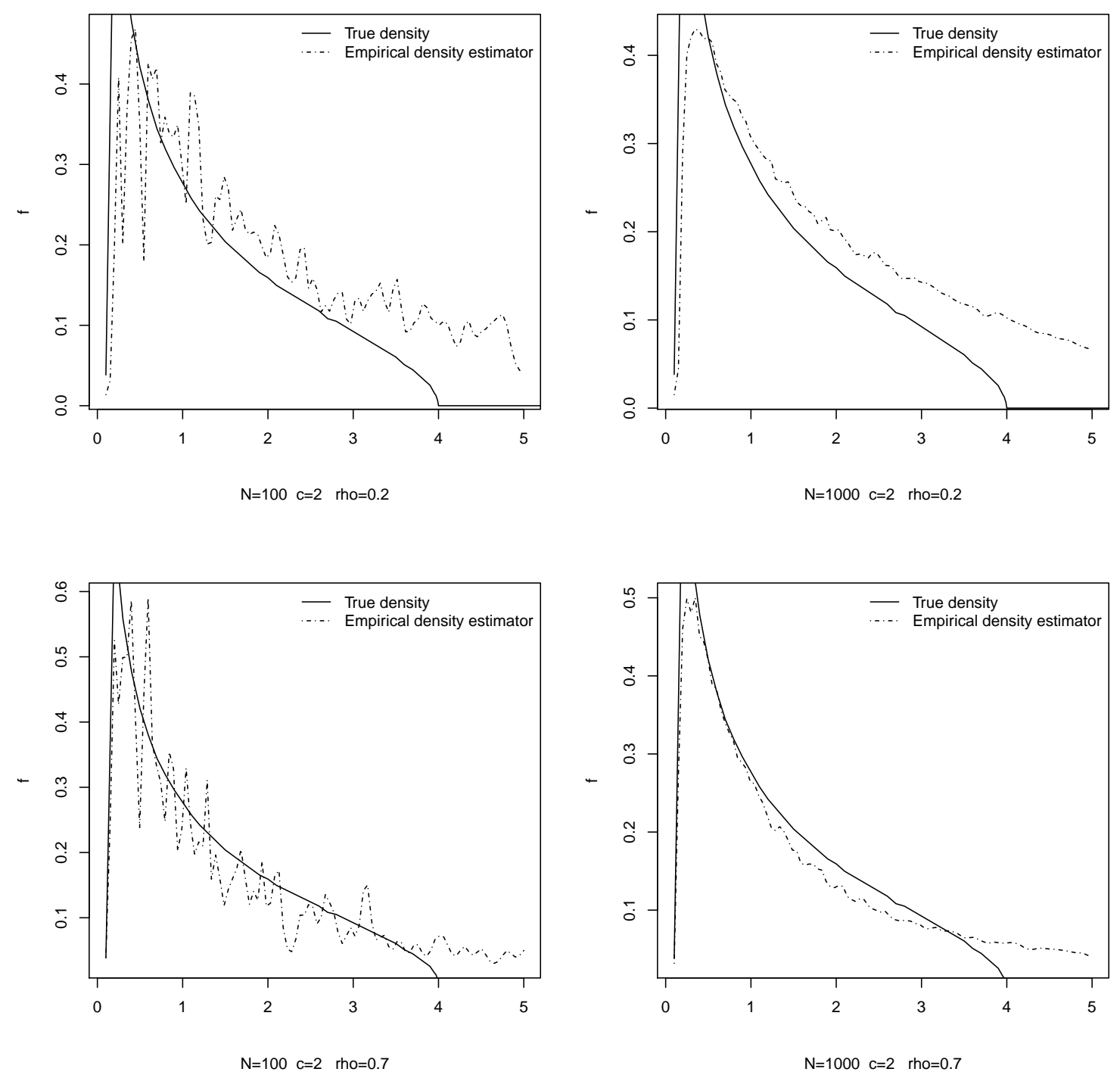

Figure 2: Densities of the limit law and empirical estimator for $c=2$ and $\rho=0.2, \rho=0.7$ with $N=100, N=1000$

Table 1: $L_{1}$-errors of the empirical density estimator in two cases: $T_{N}$ is the identity matrix with $c=1, T_{N}$ is a diagonal matrix of 3 eigenvalues.

\begin{tabular}{cccccccc}
\hline & \multicolumn{3}{c}{$\rho=0.2$} & & \multicolumn{3}{c}{$\rho=0.7$} \\
\cline { 2 - 4 } \cline { 6 - 8 } \cline { 6 - 8 } & $N=100$ & $N=500$ & $N=1000$ & & $N=100$ & $N=500$ & $N=1000$ \\
\hline Case 1 & 0.0297 & 0.0253 & 0.0200 & & 0.0390 & 0.0342 & 0.0298 \\
Case 2 & 0.0025 & 0.0020 & 0.0009 & & 0.0028 & 0.0020 & 0.0009 \\
\hline
\end{tabular}

simulate the empirical density function of the large symmetric random matrices $X_{N} T_{N} X_{N}^{t}$, in the two cases $T_{N}$ is the identity matrix $(n \times n),(n=n(N))$ and $T_{N}$ is a diagonal matrix $T_{N}=\operatorname{diag}\left(\tau_{1}, \ldots, \tau_{n}\right), \tau_{i}$ are real rv's, the LSD of $T_{N}$ is easy to determine. In the special case of the assumption (A1): $A_{N}=\rho I_{N}$ where $|\rho|<1$ and $I_{N}$ is the identity matrix of order $N$, we put ourselves for example in the case of an autoregressive linear process of order 1, AR(1). The entries $X_{i . j}$ of the matrix $X_{N}$ satisfy in each column (for each $j$ ) the AR(1) relation

$$
X_{i+1 . j}=\rho X_{i . j}+\varepsilon_{i+1 . j}, \quad i \geq 1
$$




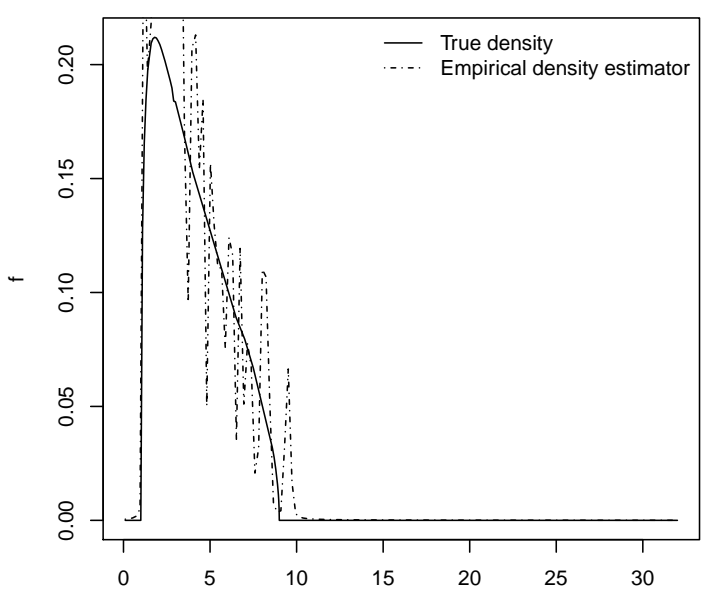

$\mathrm{N}=100 \quad \mathrm{c}=4 \quad$ rho $=0.2$

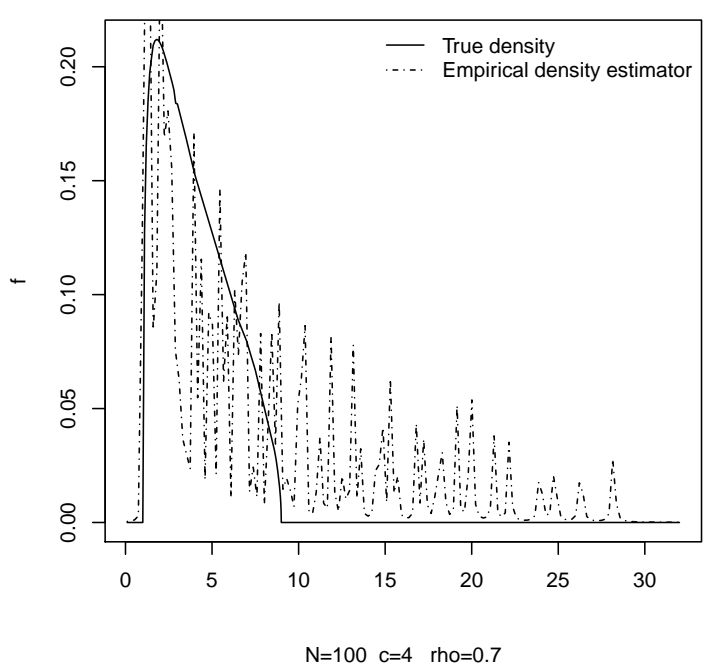

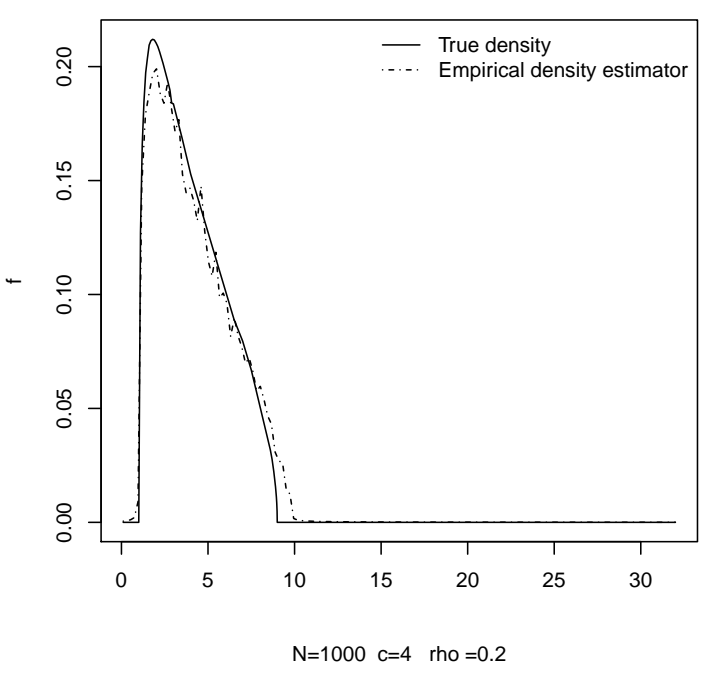

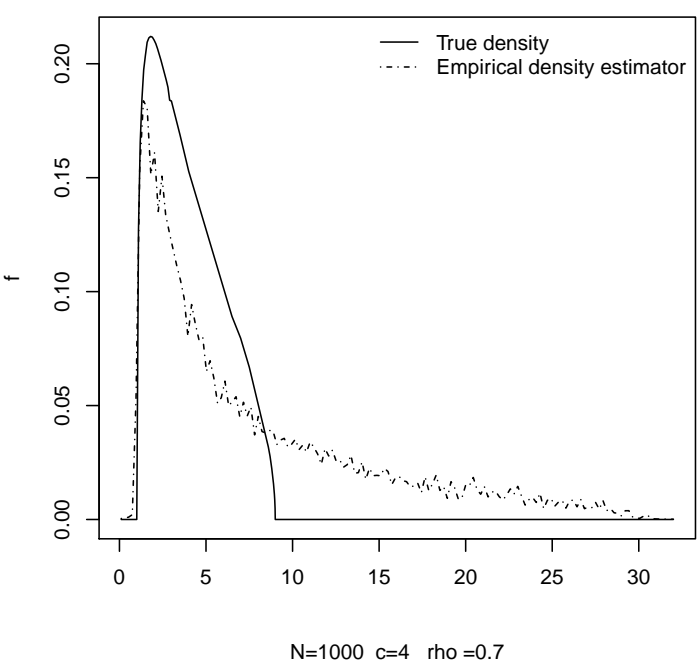

Figure 3: Densities of the limit law and empirical estimator for $c=4$ and $\rho=0.2, \rho=0.7$ with $N=100, N=1000$

where $\varepsilon_{i . j},(i=1, \ldots, N$ and $j=1, \ldots, n)$ is a white noise and $X_{1 . j}=\varepsilon_{1 . j}$. For each $j$ we draw a sample of an $\operatorname{AR}(1)$ process $X_{(j)}$ and replicate this operation for each column $j$. Afterwards, we obtain a sample of eigenvalues $\left(\lambda_{j}\right)$ of random matrix $X_{N} T_{N} X_{N}^{t}$. The Stieltjes transform

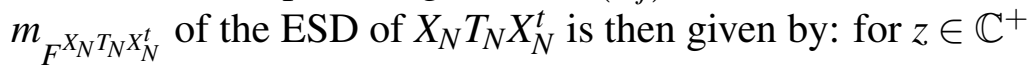

$$
m_{F^{X_{N} T_{N} X_{N}^{t}}}(z)=\frac{1}{n} \operatorname{tr}\left(X_{N} T_{N} X_{N}^{t}-z I\right)^{-1}=\frac{1}{n} \sum_{j=1}^{n}\left(\lambda_{j}-z\right)^{-1}
$$

where $t r$ denotes the trace of the matrix. From the last relation, empirical density estimator $f_{N}$ of $f$ the spectral density of $X_{N} T_{N} X_{N}^{t}$ is obtained via the formula

$$
f_{N}(x)=\frac{1}{\pi} \mathfrak{I m} m_{F^{X_{N} T_{N} X_{N}^{t}}}(z)
$$

where $z=x+i y$ and $y$ is chosen small. For the assumptions above on the matrix $T_{N}$ and the linear process $X_{(t)}$ (the $t$-th column of the matrix $X_{N}$ ), the LSD of the matrix $X_{N} T_{N} X_{N}^{t}, F$ has 

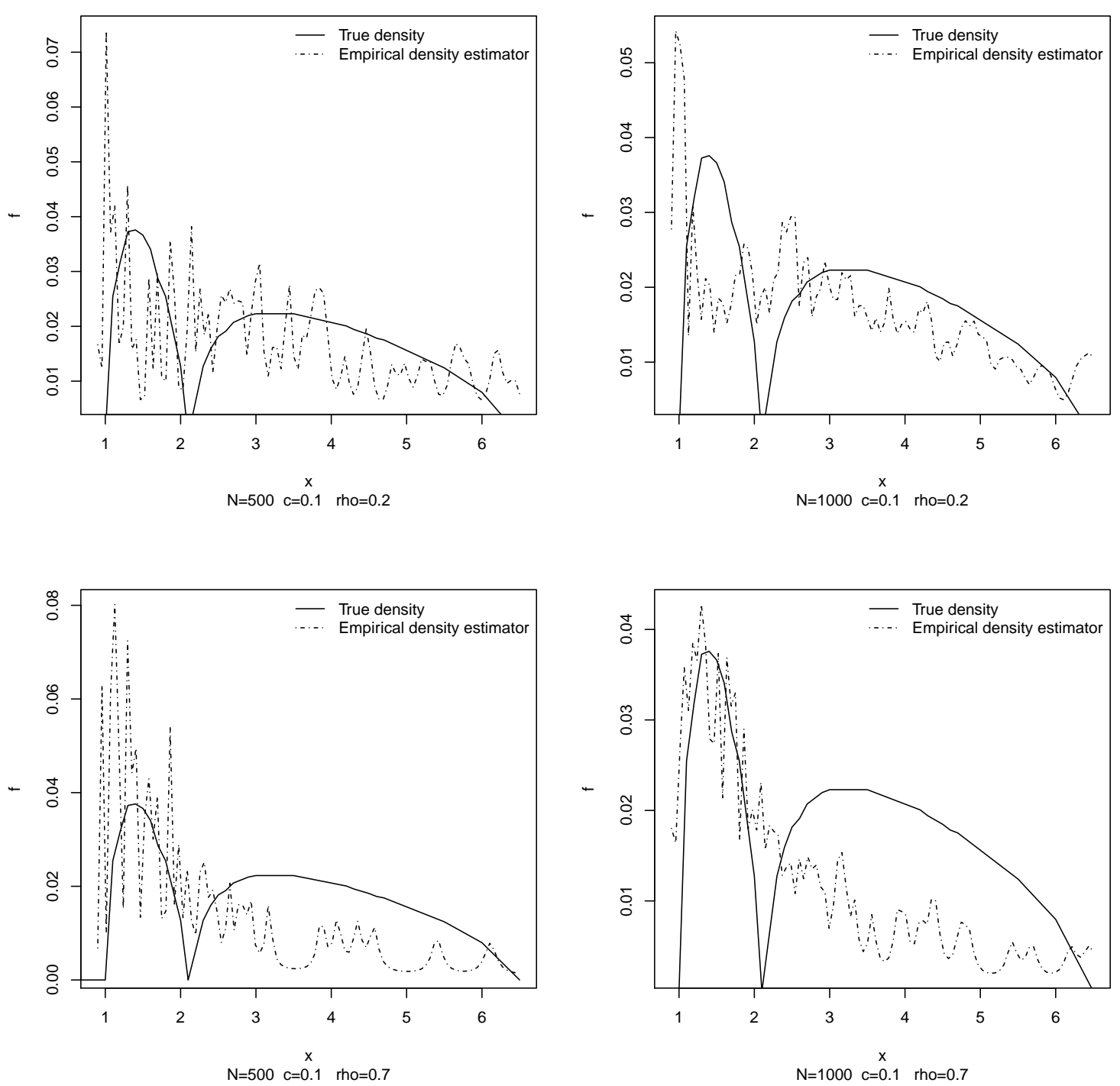

Figure 4: Densities of the limit law and empirical estimator where matrix $T_{N}$ has two eigenvalues 1.5 and 4 , for $c=0.1$ and $\rho=0.2, \rho=0.7$ with $N=500, N=1000$

a Stieltjes transform $m_{F}(z)$ given by

$$
m_{F}(z)=-\left(z-c \int \frac{\tau d H(\tau)}{1+\tau m_{F}(z)}\right)^{-1}, \quad z \in \mathbb{C}^{+} .
$$

where $H$ is the LSD of ESD of the matrix $T_{N}$. Using the relation (6), we can represent the density of the LSD of the matrix in question.

Examples are illustrated in Figure 1, Figure 2 and Figure 3 by applying the computations of the estimators by the formula (7) in the case of $T_{N}$ the identity matrix with a comparison of different values of the dimension $c$. Figure 4 provides estimators of the density in the case of $T_{N}$ diagonal matrix with two eigenvalues, the same representations are given in Figure 5 and Figure 6 with 3 and 4 eigenvalues of $T_{N}$ respectively and different choices of the other parameters.

\section{Conclusion}

Numerical simulations pointed out that in general the performance of the estimator strongly depend on the choices of the dimension $c$, parameter $\rho$ and sample size $N$. We may 

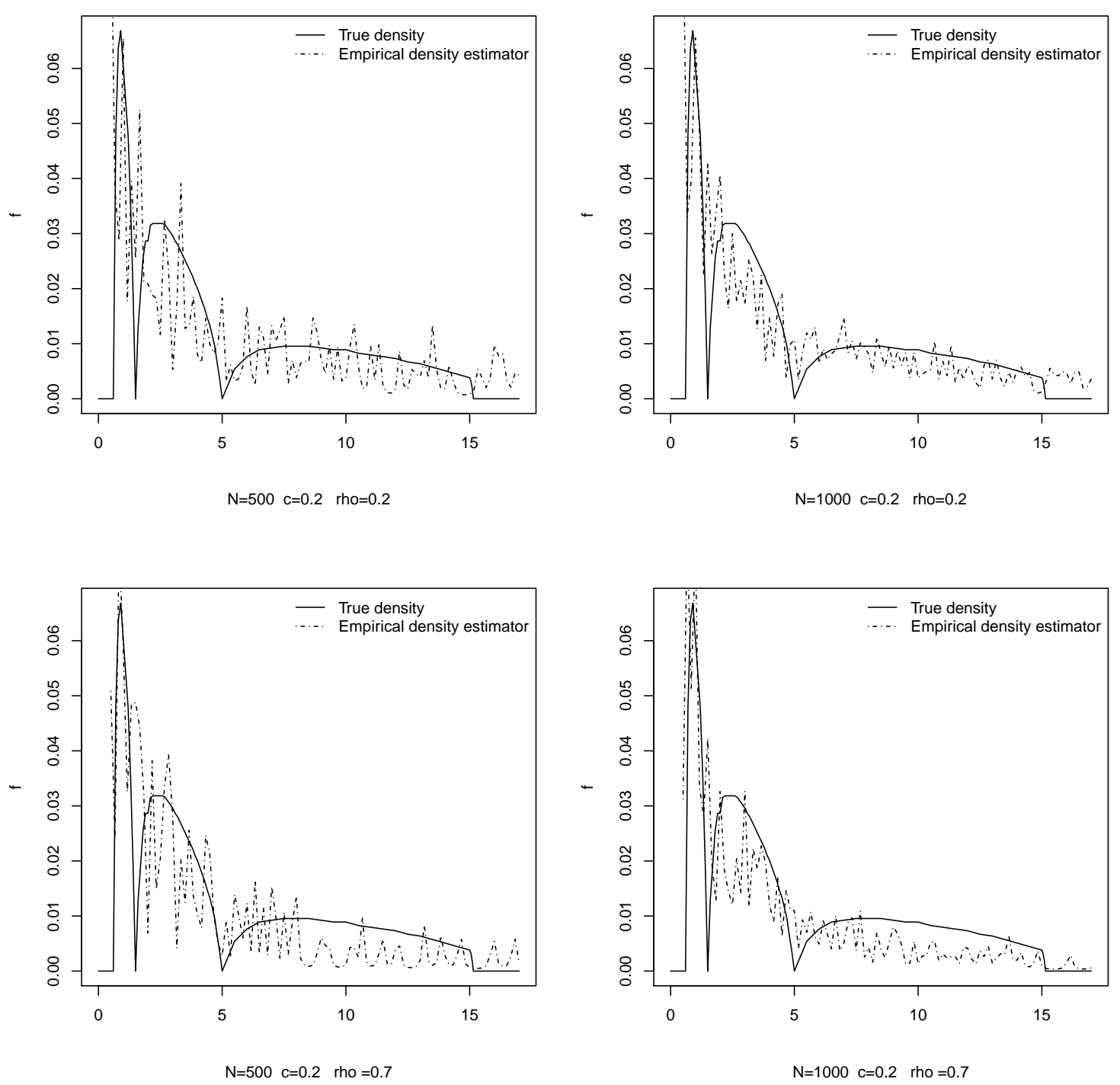

Figure 5: Densities of the limit law and empirical estimator where matrix $T_{N}$ has three eigenvalues 1,3 and 10 , for $c=0.2$ and $\rho=0.2, \rho=0.7$ with $N=500, N=1000$

observe that the variability of parameters has a direct impact on the stabilization and convergence rate of the estimator. Particular choices of parameters confirm good performance of the estimator and lead to indicate optimal values for these parameters. For weak dependence ( $\rho=0.2)$ the estimator performs quite well for moderate value $N=100$, whereas for strong dependence $(\rho=0.7)$ the estimators accurate enough only for large $N$ (when $N \geq 1000$ ). We also observe marginal influence of the dimension $c$ on density estimator convergence rate. For values of $c \geq 1$, for both weak and strong dependencies $(\rho=0.2, \rho=0.7)$ and $T_{N}$ identity matrix, the estimators perform well from on $N=100$. However for small values of $c(c \leq 0.2)$ and $T_{N}$ diagonal matrix, we observe the influence of parameter values on the convergence rate. The number of eigenvalues of $T_{N}$ has an effect on the shape of the estimator as well as on their performance, according to the graphic representation of the empirical density in the case of several eigenvalues, we notice that the curves stabilize only from a sample size $N=500$, differently in the case of only one eigenvalue where we can have a good results of estimate of the limiting density from $N=100$. Note that the choice of the values of the eigenvalues of the matrix $T_{N}$ is very important, so that if one takes eigenvalues close to one another, the curves of the empirical estimator of the density and the limit density 

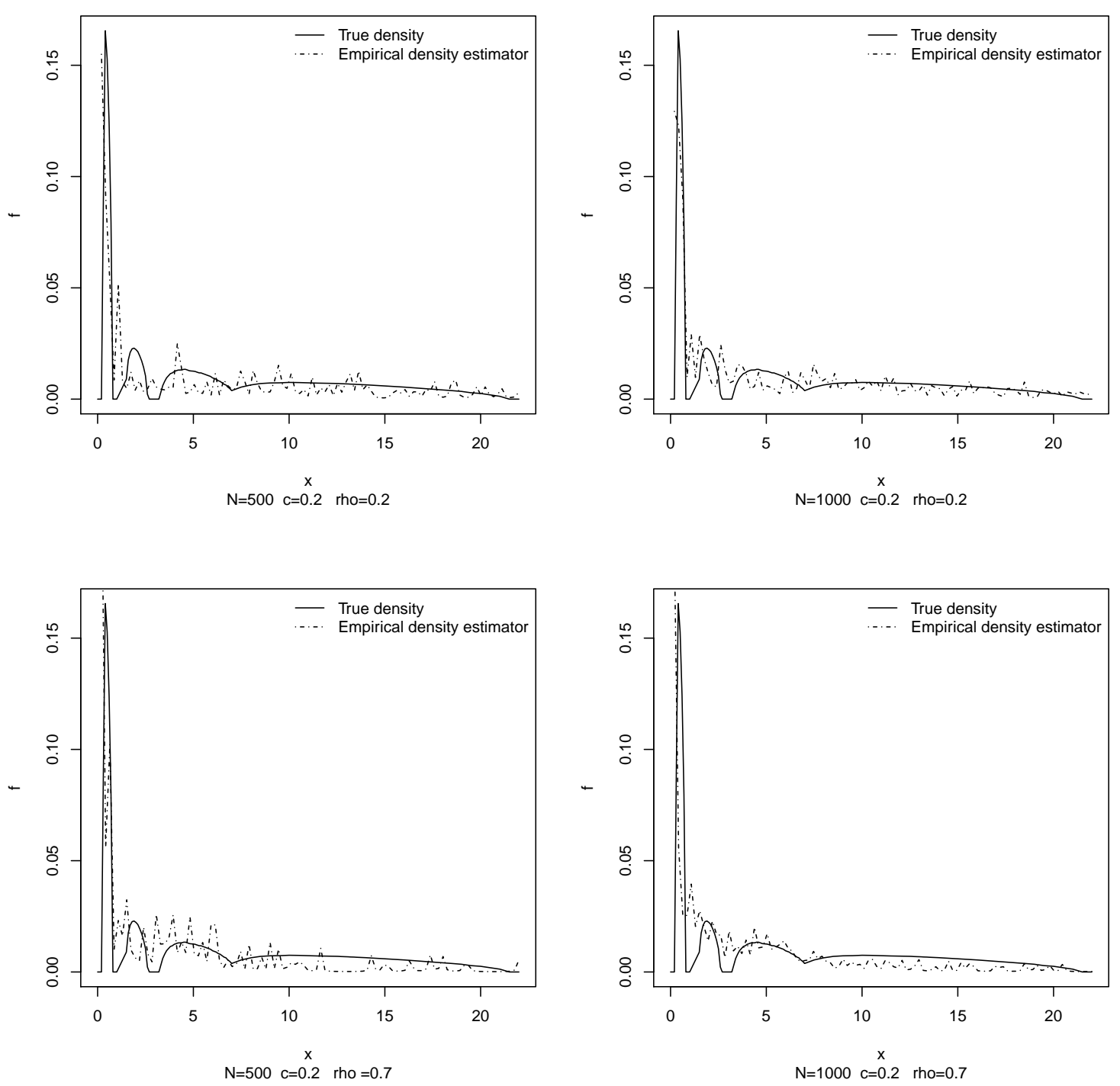

Figure 6: Densities of the limit law and empirical estimator where matrix $T_{N}$ has four eigenvalues $0.5,2,4$ and 13 , for $c=0.2$ and $\rho=0.2, \rho=0.7$ with $N=500, N=1000$

will be very close and vice versa, which leads to say that a judicious choice of simulation parameters is an essential factor to obtain the desired results.

\section{Acknowledgements}

The author is highly grateful to the two unknown reviewers for their constructive comments which led to substantial improvement in the paper.

\section{References}

[1] Bai, Z. D. and Silverstein, J. W. (2010): Spectral analysis of large dimensional random matrices. New York, NY: Springer.

[2] Bai, Z. D. and Silverstein, J. W. (1999): Exact separation of eigenvalues of large dimentional random matrices. Annals of Probability, 27, 1536-1555.

[3] Bhattacharjee, M. and Bose, A. (2016): Large sample behaviour of high dimensional autocovariance matrices. The Annals of Statistics, 44, 598-628. 
[4] Boutet de Monvel, A. and Khorunzhy, A. (1998): Limit theorems for random matrices with correlated entries. Markov Process and Related Fields, 4, 175-197.

[5] Grenander, U. and Silverstein, J. W. (1977): Spectral analysis of networks with random topologies. SIAM Journal on Applied Mathematics, 32, 499-519.

[6] Jin, B., Wang, C., Bai, Z. D., Nair, K., and Harding, M. (2014): Limiting spectral distribution of a symmetrized auto-cross covariance matrix. Annals of Applied Probability 24, 1199-1225.

[7] Jonsson, D. (1982): Some limit theorems for the eigenvalues of a sample covariance matrix. Journal of Multivariate Analysis, 12, 1-38.

[8] Marcenko, V. A. and Pastur, L. A. (1967): Distribution of eigenvalues in certain sets of random matrices. Mathematics of the USSR-Sbornik, 72, 507-536.

[9] Merlevede, F. and Peligrad, M. (2016): On the empirical spectral distribution for matrices with long memory and independent rows. Stochastic Processes and their Applications, 126, 2734-2760.

[10] Silverstein, J. W.(1995): Strong convergence of the empirical distribution of eigenvalues of large dimentional random matrices. Journal of Multivariate Analysis, 54, $175-192$.

[11] Silverstein, J. W. (2009): The Stieltjes transform and its role in eigenvalue behavior of large dimensional random matrices. Random Matrix Theory and its Applications, 18; $1-25$.

[12] Silverstein, J. W. and Bai, Z. D. (1995): On the empirical distribution of eigenvalues of a class of large dimensional random matrices. Journal of Multivariate Analysis, $\mathbf{5 4}$, $175-192$.

[13] Silverstein, J. W. and Choi, S. I. (1995): Analysis of the limiting spectral distribution of large dimensional random matrices. Journal of Multivariate Analysis, 54, 295-309.

[14] Wachter, K. W. (1980): The limiting empirical measure of multiple discriminant ratios. The Annals of Statistics, 8, 937-957.

[15] Wang, L. and Paul, D. (2014): Limiting spectral distribution of renormalized separable sample covariance matrices when $p / n \rightarrow 0$. Journal of Multivariate Analysis, 126, 2552.

[16] Yin, Y. Q. (1986): Limiting spectral distribution for a class of random matrices. Journal of Multivariate Analysis, 20, 50-68. 